Mathematical Sciences And Applications E-Notes

Volume 3 No. 2 PP. 27-33 (2015) @ MSAEN

\title{
ON TRIGONOMETRIC FUNCTIONS AND NORM IN THE GENERALIZED TAXICAB METRIC
}

\author{
S. EKMEKÇI, Z. AKÇA AND A. K. ALTINTAŞ \\ (Communicated by Bayram ŞAHIN)
}

\begin{abstract}
In this paper, the plane with the generalized taxicab metric is considered and the trigonometric functions and the norm are defined. Then, the cosine and the sine functions are developed by using the reference angle with respect to the generalized taxicab metric. It is shown that Schwarz's inequality is valid under restricted case of $d_{T_{g}}$-metric. Finally, the area of any triangle in the plane with the generalized taxicab metric is given as the geometrical interpretation that contains the trigonometric functions and norm.
\end{abstract}

\section{INTRODUCTION}

The Minkowski metric of order $k(k \geq 1)$ is for distance between two points $A=\left(x_{1}, y_{1}\right)$ and $B=\left(x_{2}, y_{2}\right)$ determined by

$$
d_{k}(A, B)=\left(\left|x_{2}-x_{1}\right|^{k}+\left|y_{2}-y_{1}\right|^{k}\right)^{\frac{1}{k}} .
$$

Minkowski metric family include taxicab metric, $d_{T}$, for $k=1$ and Euclidean metric, $d_{E}$, for $k=2$. Taxicab geometry was introduced by Menger [11] and developed by Krause [10]. Taxicab geometry has been studied and developed by some mathematicians. The taxicab plane, $\mathbb{R}_{T}^{2}$, is almost the same as the Euclidean plane $\mathbb{R}^{2}$. The points and lines are the same, and the angles are measured the same way, but the distance function is different. The unit circle with the equation $|x|+|y|=1$ is a square with oriented at $45^{\circ}$ angle to the coordinate axes in $\mathbb{R}_{T}^{2}$ for all points $(x, y)$.

In [14], Lawrence J. Wallen altered taxicab distance by redefining in order to get rid of possibly misleading symmetry. For two points $A=\left(x_{1}, y_{1}\right)$ and $B=\left(x_{2}, y_{2}\right)$ in $\mathbb{R}^{2}$, the (slightly generalized) taxicab distance function is defined $d_{T_{g}}(A, B)=$ $a\left|x_{2}-x_{1}\right|+b\left|y_{2}-y_{1}\right|$, where $a, b>0$. Also, it is shown that the distance $d_{T_{g}}$ determines a metric. Note that the generalized taxicab metric is the taxicab metric, when $a=b=1$. The analytical plane with the distance $d_{T_{g}}$ will be denoted by $\mathbb{R}_{T_{g}}^{2}$. In $\mathbb{R}_{T_{g}}^{2}$, the unit circle with the equation $a|x|+b|y|=1$ is a diamond that

Date: Received: March 12, 2015; Revised: June 05, 2015; Accepted: August 26, 2015.

2010 Mathematics Subject Classification. 51K05,51K99.

Key words and phrases. trigonometric functions, non- Euclidean metric, generalized taxicab metric. 
its vertex vectors are $v_{1}=\left(\frac{1}{a}, 0\right), v_{2}=\left(0, \frac{1}{b}\right), v_{3}=\left(-\frac{1}{a}, 0\right)$ and $v_{4}=\left(0,-\frac{1}{b}\right)$. The shortest distance $d_{T_{g}}$ between the points $A$ and $B$ is the union of the line segments with the same slopes as $v_{i}$ and $v_{i+1}, i=\{1,2,3,4\}$, when the vector $A B$ is in the sector obtained by extending the vectors $v_{i}$ and $v_{i+1}$. If the slope of the line segment $A B$ is equal to the slope of $v_{i}, i=\{1,2,3,4\}$, then the $d_{T_{g}}$-distance of $A B$ is constant $a$ or $b$ multiple of the Euclidean distance between $A$ and $B,[3]$. Also,the group of isometries of the plane with generalized taxicab metric is studied in [9].

The unit circle has a different point set and a different figure in different metrics. So, the values of trigonometric functions change according to the metric which we use. The trigonometric functions on the unit circle of Taxicab, the Chinese Checker, the Maximum metrics and the Generalized Absolute metrics have been defined and developed in $[2,4,5,6,7,8,13]$.

In the present paper, the trigonometric functions are defined using the generalized taxicab metric. And, several trigonometric identities of these functions are given. Also, the definitions of trigonometric functions are developed by using the reference angle and the change of the generalized taxicab length of a line segment after rotations is studied. Later on, the norm is introduced using trigonometric functions and the Schwarz's inequality is looked at. Finally, the area of any triangle is given by using norm and trigonometric functions in the plane with generalized taxicab metric.

\section{Trigonometric Functions in $\mathbb{R}_{T_{g}}^{2}$}

We want to give the standart definitions of the trigonometric functions on unit circle in $\mathbb{R}_{T_{g}}^{2}$ as in $[4,6,7,8]$. In plane geometry, trigonometric functions are defined as $x=\cos \theta, y=\sin \theta$ for all points $(x, y)$ on the unit circle, where $\theta$ is the angle with initial side the positive $x$-axis and terminal side the radial line passing through point $(x, y)$. Since the slope of radial line passing through point $(x, y)$ does not change, tangent function does not depend on the metric. So, the trigonometric functions can be defined according to the generalized taxicab metric in terms of the standard Euclidean tangent function. Thus, the slope of the radial line goes through the point $\left(\cos _{T_{g}} \theta, \sin _{T_{g}} \theta\right)$ on the unit circle in $\mathbb{R}_{T_{g}}^{2}$ is

$$
\tan \theta=\frac{\sin _{T_{g}} \theta}{\cos _{T_{g}} \theta}=\tan _{T_{g}} \theta .
$$

For the definitions of sine and cosine, it is necessary to find only the point that is called $\left(\cos _{T_{g}} \theta, \sin _{T_{g}} \theta\right)$ is on the line that makes an angle $\theta$ with the positive $x$-axis and on the unit circle in $\mathbb{R}_{T_{g}}^{2}$. The equation of the line joining $(x, y)$ and $(0,0)$ is $y=(\tan \theta) x$. Solving the system

$$
\left\{\begin{array}{l}
y=(\tan \theta) x \\
a|x|+b|y|=1
\end{array}\right.
$$

we have the following chain of results

$$
\begin{aligned}
& |x|=\frac{1}{a+b|\tan \theta|}=\left|\cos _{T_{g}} \theta\right| \\
& |y|=\frac{|\tan \theta|}{a+b|\tan \theta|}=\left|\sin _{T_{g}} \theta\right| .
\end{aligned}
$$


If it is made appropriate choice of sign for the absolute value based on the quadrant, cosine and sine functions in $\mathbb{R}_{T_{g}}^{2}$ are given as follows

$$
\begin{aligned}
\cos _{T_{g}} \theta & =\frac{\cos \theta}{a|\cos \theta|+b|\sin \theta|} \\
\sin _{T_{g}} \theta & =\frac{\sin \theta}{a|\cos \theta|+b|\sin \theta|}
\end{aligned}
$$

The graphs of cosine and sine functions in $\mathbb{R}_{T_{g}}^{2}$ are presented in the following figures.

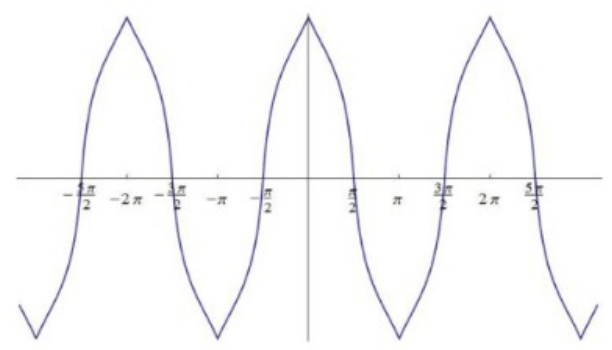

FiguRE 1. $y=\cos _{T_{g}} x$ for $a=5, b=2$

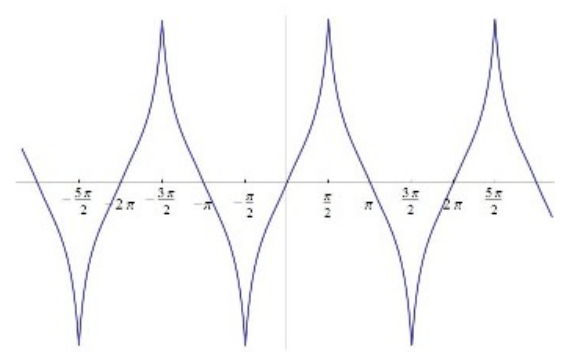

FiguRE 2. $y=\sin _{T_{g}} x$ for $a=5, b=2$

In $\mathbb{R}_{T_{g}}^{2}$, the trigonometric identities will differ from their Euclidean analogues in most cases. If the secant and cosecant functions are defined as in Euclidean plane, then $\csc _{T_{g}} \theta=\frac{1}{\sin _{T_{g}} \theta}, \sec _{T_{g}} \theta=\frac{1}{\cos _{T_{g}} \theta}$. The cotangent function is not based on the generalized taxicab metric as tangent function. The cofunction identities of these functions are similar to their Euclidean analogues. That is, $\cos _{T_{g}}\left(\frac{\pi}{2}-\theta\right)=\sin _{T_{g}} \theta$ and $\sin _{T_{g}}\left(\frac{\pi}{2}-\theta\right)=\cos _{T_{g}} \theta$.

Using the identities $\tan (-\theta)=-\tan \theta$ and $\cot (-\theta)=-\cot \theta$ in (2.2) and (2.3), one can verify the identities $\cos _{T_{g}}(-\theta)=\cos _{T_{g}} \theta$ and $\sin _{T_{g}}(-\theta)=-\sin _{T_{g}} \theta$.

It is well known that the Pythagorean identity is the relation between sine and cosine functions. In the meaning of the generalized taxicab metric, the Pythagorean identities are obtained by using unit circle in $\mathbb{R}_{T_{g}}^{2}$ as follows:

$$
a\left|\cos _{T_{g}} \theta\right|+b\left|\sin _{T_{g}} \theta\right|=1,
$$




$$
\begin{aligned}
& a+b|\tan \theta|=\left|\sec _{T_{g}} \theta\right|, \\
& a|\cot \theta|+b=\left|\csc _{T_{g}} \theta\right| .
\end{aligned}
$$

Unlike the Euclidean case, there is a non-uniform change in arc length as the angle $\theta$ is increased by a fixed amount in the generalized taxicab metric. So, it is necessary to develop the trigonometric functions defined on the unit circle in $\mathbb{R}_{T_{g}}^{2}$ for any angle $\theta$ using the reference angle $\alpha$ of $\theta$ [13].

Definition 2.1. Let $\theta$ be the angle with the reference angle $\alpha$ which is the angle between $\theta$ and the positive direction of the $x$ - axis on unit circle in $\mathbb{R}_{T_{g}}^{2}$. The cosine and sine functions of the angle $\theta$ with the reference angle $\alpha, T_{g} \cos \theta$ and $T_{g} \sin \theta$, are defined as

$$
\begin{aligned}
& T_{g} \cos \theta=\cos _{T_{g}}(\alpha+\theta) \cdot \cos _{T_{g}} \alpha+\sin _{T_{g}}(\alpha+\theta) \cdot \sin _{T_{g}} \alpha \\
& T_{g} \sin \theta=\sin _{T_{g}}(\alpha+\theta) \cdot \cos _{T_{g}} \alpha-\cos _{T_{g}}(\alpha+\theta) \cdot \sin _{T_{g}} \alpha .
\end{aligned}
$$

One can easily see that $T_{g} \cos \theta=\frac{1}{a} \cos _{T_{g}} \theta$ and $T_{g} \sin \theta=\frac{1}{a} \sin _{T_{g}} \theta$ for $\alpha=0$.

Consequently, the general definitions of trigonometric functions can be given by defining angles with the reference angle in the generalized taxicab metric.

\section{The Length of a Line Segment Under Rotations in $\mathbb{R}_{T_{g}}^{2}$}

It is well known that all rotations, translations and reflections preserve the Euclidean distance. But the length of a line segment under rotations changes in non-Euclidean metrics as in [12]. In $\mathbb{R}_{T_{g}}^{2}$, all translations and the rotations with the angle $\theta \in\{0, \pi\}$ for $a \neq b$ or $\theta \in\left\{0, \frac{\pi}{2}, \pi, \frac{3 \pi}{2}\right\}$ for $a=b$ preserve $d_{T_{g}}$-distance [9]. Also, $d_{T_{g}}(l) \neq d_{E}(l)$ for $a^{2}+b^{2} \neq 1$ and $m \neq \pm \frac{b}{a}$ such that $m$ is the slope of the line segment $l$. Thus, the change of a line segment length after rotation is given by the following theorem:

Theorem 3.1. Let any two points be $A$ and $B$ in $\mathbb{R}_{T_{g}}^{2}$, and let the line segment $A B$ be not parallel to the $x$-axis and $\alpha$ be the angle between the line segment $A B$ and the positive direction of $x$-axis. If $A^{\prime} B^{\prime}$ is the image of $A B$ under the rotation with the angle $\theta$, then $d_{T_{g}}\left(A^{\prime}, B^{\prime}\right)$ is equal to

$d_{T_{g}}(A, B) \sqrt{\frac{\cos _{T_{g}}^{2} \alpha+\sin _{T_{g}}^{2} \alpha}{\cos _{T_{g}}^{2}(\alpha+\theta)+\sin _{T_{g}}^{2}(\alpha+\theta)}}=d_{T_{g}}(A, B) \frac{a|\cos (\alpha+\theta)|+b|\sin (\alpha+\theta)|}{a|\cos \alpha|+b|\sin \alpha|}$.

Proof. Since all the translations are preserve in $d_{T_{g}}$-distance, the line segment $A B$ can be translated to line segment $O X$ such that $d_{T_{g}}(A, B)=d_{T_{g}}(O, X)=k$. Let the line segment $O X^{\prime}$ be the image of $O X$ under rotation with the angle $\theta$. If $\alpha$ is the reference angle of $\theta$, then $X=\left(k \cos _{T_{g}} \alpha, k \sin _{T_{g}} \alpha\right)$. Let $d_{T_{g}}\left(O, X^{\prime}\right)$ be $k^{\prime}$. Then $X^{\prime}=\left(k^{\prime} \cos _{T_{g}}(\alpha+\theta), k^{\prime} \sin _{T_{g}}(\alpha+\theta)\right)$. Using the equality of Euclidean lengths of the line segments $O X$ and $O X^{\prime}$ one get

$d_{T_{g}}\left(A^{\prime}, B^{\prime}\right)=d_{T_{g}}\left(O, X^{\prime}\right)=k \sqrt{\frac{\cos _{T_{g}}^{2} \alpha+\sin _{T_{g}}^{2} \alpha}{\cos _{T_{g}}^{2}(\alpha+\theta)+\sin _{T_{g}}^{2}(\alpha+\theta)}}=k \frac{a|\cos (\alpha+\theta)|+b|\sin (\alpha+\theta)|}{a \mid \cos \alpha)|+b| \sin \alpha \mid}$.

The following corollary shows how one can find the the generalized taxicab length, after a rotation of a line segment with an angle $\theta$ in standard form. 
ON TRIGONOMETRIC FUNCTIONS AND NORM IN THE GENERALIZED TAXICAB... 31

Corollary 3.1. Let the line segment $A B$ is parallel to the $x$-axis. If $A^{\prime} B^{\prime}$ is the image of $A B$ under the rotation with an angle $\theta$ then

$$
d_{T_{g}}\left(A^{\prime}, B^{\prime}\right)=\frac{d_{T_{g}}(A, B)}{\sqrt{\cos _{T_{g}}^{2} \theta+\sin _{T_{g}}^{2} \theta}} .
$$

Proof. Using the value $\alpha=0$ in the previous theorem, the corollary is obtained.

\section{The Norm IN $\mathbb{R}_{T_{g}}^{2}$}

In [14], According to $d_{T_{g}}$, the norm of a vector $v=\overrightarrow{O P}$ ( $O$ is the origin) is defined by

$$
\|v\|_{T_{g}}=d_{T_{g}}(O, P)=a|x|+b|y|,
$$

where $v=(x, y)$ in $\mathbb{R}^{2}$. If the vector $v$ lies in the sector determined by $v_{i}$ and $v_{i+1}$, then $\|v\|_{T_{g}}=t_{i}+t_{i+1}$ where $t_{i}, t_{i+1}$ are nonnegative real numbers such that $v=t_{i} v_{i}+t_{i+1} v_{i+1}$. Now, it will be given that the norm can be stated by using the standard inner product and trigonometric functions in $\mathbb{R}_{T_{g}}^{2}$.

Definition 4.1. Let $v=(x, y)$ be any vector in $\mathbb{R}_{T_{q}}^{2}$. Then the norm of $v$ is defined by

$$
\|v\|_{T_{g}}=\sqrt{\frac{\langle v, v\rangle}{\cos _{T_{g}}^{2} \alpha+\sin _{T_{g}}^{2} \alpha}},
$$

where $\alpha$ is the reference angle of $v$.

Corollary 4.1. $\|v\|_{T_{g}}=d_{T_{g}}(O, v)$.

Proof. If $v=(x, y)=\left(d_{T_{g}}(O, v) \cos _{T_{g}} \alpha, d_{T_{g}}(O, v) \sin _{T_{g}} \alpha\right)$ is used where $\alpha$ is the reference angle of $v$,

$$
d_{E}(O, v)=\sqrt{\cos _{T_{g}}^{2} \alpha+\sin _{T_{g}}^{2} \alpha} d_{T_{g}}(O, v) .
$$

If $d_{E}(O, v)=\sqrt{\langle v, v\rangle}$ and above definition are used,

$$
\|v\|_{T_{g}}=d_{T_{g}}(O, v)
$$

is obtained.

Also, If the vector $v$ lies in the sector determined by $v_{i}$ and $v_{i+1}$, then $\|v\|_{T_{g}}=$ $t_{i}+t_{i+1}$ where $t_{i}, t_{i+1}$ are nonnegative real numbers such that $v=t_{i} v_{i}+t_{i+1} v_{i+1}$. From Corollary (4.1) and Definition (4.1),

$$
\|v\|_{T_{g}}=d_{T_{g}}(O, v)=t_{i}+t_{i+1}=\sqrt{\frac{\langle v, v\rangle}{\cos _{T_{g}}^{2} \alpha+\sin _{T_{g}}^{2} \alpha}} .
$$

As in Euclidean geometry, it is easily seen that the norm function $\|\cdot\|_{T_{g}}$ satisfies the following properties. So, it is given without proof.

Proposition 4.1. Let $u=\left(x_{1}, y_{1}\right), v=\left(x_{2}, y_{2}\right)$ and $w=\left(x_{3}, y_{3}\right)$ be any three vectors in $\mathbb{R}_{T_{g}}^{2}$ and $r \in \mathbb{R}$. Then 
(i) $\|v\|_{T_{q}} \geq 0$ and $\|v\|_{T_{g}}=0 \Leftrightarrow v=0$,

(ii) $\|r v\|_{T_{g}}=|r| \cdot\|v\|_{T_{g}}$,

(iii) $\|v+w\|_{T_{g}} \leq\|v\|_{T_{g}}+\|w\|_{T_{g}}$,

(iv) $\|v-w\|_{T_{g}} \geq\|v\|_{T_{g}}-\|w\|_{T_{g}}$,

(v) $\|v-w\|_{T_{g}} \geq\|v-u\|_{T_{g}}-\|u-w\|_{T_{g}}$.

It is very well known that Schwarz's inequality is an important property shared by inner product and the norm. Now, it is shown that Schwarz's inequality is valid for some restricted cases of $d_{T_{g}}$ in $\mathbb{R}_{T_{g}}^{2}$.

Proposition 4.2. (Schwarz's inequality) If $v=\left(x_{1}, y_{1}\right)$ and $w=\left(x_{2}, y_{2}\right)$ be any two vectors in $\mathbb{R}_{T_{g}}^{2}$ and $a \geq 1, b \geq 1$, then

$$
|\langle v, w\rangle| \leq\|v\|_{T_{g}}\|w\|_{T_{g}} .
$$

Proof. If $v=0$, then $|\langle v, w\rangle|=0$ and $\|v\|_{T_{g}}=0$. The equality holds. Let $v \neq 0$, it is known that

$$
\begin{gathered}
|\langle v, w\rangle|=\left|x_{1} x_{2}+y_{1} y_{2}\right|, \\
\|v\|_{T_{g}}\|w\|_{T_{g}}=\left(a\left|x_{1}\right|+b\left|y_{1}\right|\right)\left(a\left|x_{2}\right|+b\left|y_{2}\right|\right)
\end{gathered}
$$

If the right-hand sides of (4.1) and (4.2) are observed,

$$
a b\left|x_{1} y_{2}\right|+a b\left|x_{2} y_{1}\right|+a^{2}\left|x_{1} x_{2}\right|+b^{2}\left|y_{1} y_{2}\right| \geq\left|x_{1} x_{2}+y_{1} y_{2}\right|
$$

is valid when $a \geq 1, b \geq 1$. This completes the proof.

In the following theorem, the area of the triangle $A \vec{B} C$ is given by using the norm and trigonometric functions in $\mathbb{R}_{T_{g}}^{2}$.

Theorem 4.1. Let $A B \vec{B}$ and $\theta$ be any triangle in $\mathbb{R}_{T_{g}}^{2}$ and the angle between $A C$ and $B C$. Then the area $\mathcal{A}$ of the triangle $A \vec{B} C$ is

$$
\mathcal{A}=\frac{1}{2}\|A C\|_{T_{g}}\|B C\|_{T_{g}} T_{g} \sin \theta .
$$

Proof. Since all the translations preserve $d_{T_{g}}$-distance, the point $C$ can be taken the origin. One can obtain the following relation between $T_{g} \sin \theta$ and $\sin \theta$

$$
T_{g} \sin \theta=\frac{\|O A\|\|O B\|}{\|O A\|_{T_{g}}\|O B\|_{T_{g}}} \sin \theta .
$$

From (4.3) and $2 \mathcal{A}=\|A C\|\|B C\| \sin \theta$ for $0 \leq \theta \leq \pi, \mathcal{A}=\frac{1}{2}\|A C\|_{T_{g}}\|B C\|_{T_{g}} T_{g} \sin \theta$.

\section{REFERENCES}

[1] Akça, Z., Bayar, A. and Ekmekçi, S., The norm in CC-plane geometry. Pi Mu Epsilon J. 12 (2007), no. 6, 321-324.

[2] Akça, Z. and Kaya, R., On Taxicab Trigonometry. Jour. of Inst. of Math \& Comp. Sci. (Math. Ser.) 10 (1997), no. 3, 151-159 .

[3] Altıntaş, A.K., Öklidyen Düzlemdeki Bazı Geometrik Problemlerin Genelleştirilmiş Taksi Metrikli Geometriye Uygulaması. Eskişehir Osmangazi Üniversitesi, Fen Bilimleri Enstitüsü, Yüksek Lisans Tezi, 2009.

[4] Bayar, A., On Trigonometric Functions in Maximum Metric. Kog 12 (2008), 45-48 . 
ON TRIGONOMETRIC FUNCTIONS AND NORM IN THE GENERALIZED TAXICAB... 33

[5] Bayar, A. , Ekmekçi, S., Özcan, M., On Trigonometric Functions and Cosine and Sine Rules in Taxicab Plane. International Electronic Journal Of Geometry 2 (2009), no. 1, 17-24 .

[6] Bayar, A., Ekmekçi, S. and Akça, Z., On the plane geometry with generalized absolute value metric. Mathematical Problems in Engineering Vol.2008, Article ID 673275, 8 pages.

[7] Bayar, A. and Ekmekçi, S., On the Chinese-Checker Sine And Cosine Functions. International Journal of Mathematics and Analysis 1 (2006), no. 3, 249-259 .

[8] Brisbin, R. and Artola, P., Taxicab Trigonometry. Pi Mu Epsilon J. 8 (1985), no. 2, 89-95 .

[9] Ekmekçi, S., Bayar, A., Altıntaş, A. K., On the Group of Isometries of the Generalized Taxicab Plane. International Journal of Comtemporary Mathematical Sciences 10 (2015), no. 4, 159-166 .

[10] Krause, E.F., Taxicab Geometry, Addison - Wesley Publishing Company, Menlo Park, CA (1975).

[11] Menger, K.,You Will Like Geometry, Guidebook of the Illinois Institute of Technology Geometry Exhibit, Museum of Science and Industry, Chicago, Illinois, 1952.

[12] Özcan, M., Ekmekçi, S. and Bayar, A., A Note on the Variation of the Taxicab Lengths Under Rotations. Pi Mu Epsilon J. 11 (2002), no. 7, 381-384 .

[13] Thompson, K. and Dray, T., Taxicab Angles and Trigonometry. Pi Mu Epsilon J. 11 (2000), no. $2,87-97$.

[14] Wallen, L. J., Kepler, The Taxicab Metric, and Beyond: An isoperimetric Primer. The College Mathematics Journal 26 (1995), no. 3, 178-190.

Eskişehir Osmangazi University, Department of Mathematics and Computer Science, 26480 ESKIŞEHIR, TURKEY.

E-mail address: sekmekci@ogu.edu.tr 